\title{
ASPECTOS SOCIODEMOGRÁFICOS, HISTÓRIA SEXUAL REPRODUTIVA E CONHECIMENTO CONTRACEPTIVO DE PUÉRPERAS ADOLESCENTES E ADULTAS
}

\section{SOCIO-DEMOGRAPHIC ASPECTS, SEXUAL REPRODUCTIVE HISTORY AND CONTRACEPTIVE KNOWLEDGE OF PUERPERAL TEENAGERS AND ADULTS}

\section{CARACTERÍSTICAS SOCIODEMOGRÁFICAS, HISTORIA REPRODUCTIVA SEXUAL Y EL CONOCIMIENTO DE ANTICONCEPTIVOS DE ADOLESCENTES Y ADULTOS}

Maristela Oliveira Lara ${ }^{1}$, Renata Caroline Ribeiro Lima², Luciana Angélica Vieira Santos ${ }^{2}$, André Freire Rocha ${ }^{3}$, Euza Mara Rocha ${ }^{4}$ Gabriela de Cássia Ribeiro 5

\section{RESUMO}

Objetivo: descrever e comparar os aspectos sociodemográficos, a história sexual e reprodutiva e o conhecimento contraceptivo de puérperas adolescentes e adultas que tiveram seus partos numa maternidade de referência para a macrorregião de saúde. Métodos: foi realizada entrevista individual com um instrumento semiestruturado contendo questões relativas aos aspectos estudados. Resultados: a média de idade das puérperas foi de 26,74 anos. Dentre os métodos contraceptivos, a pílula foi apontada como mais conhecido. Houve associação estatisticamente significante entre o período fértil e a idade da puérpera e a renda. A mediana de idade em que as puérperas tiveram sua primeira relação sexual foi de 17 anos. A associação idade da puérpera e idade da primeira relação sexual $(p<0,001)$ identificou início mais cedo da atividade sexual entre as adolescentes. Conclusão: as puérperas, em geral, possuem conhecimento inadequado do período fértil, além do conhecimento limitado acerca dos métodos contraceptivos. Há necessidade de maior atenção à assistência e ao planejamento familiar.

Descritores: Gravidez; Comportamento sexual; História reprodutiva; Planejamento familiar; Anticoncepção.

\begin{abstract}
Objective: to describe and to compare the sociodemographic aspects, the sexual and reproductive history and contraceptive knowledge of postpartum teenagers and adults who had their births in a reference maternity for the health macro-region. Methods: we used individual interview with a semi-structured instrument containing questions related to the previously studied aspects. Results: the average of the mothers' age was 26.74 years old. Among the methods of contraception, the pill was the most known by the mothers. There was a statistically significant association between the fertile phase, the age of puerperal woman and income. The median age at which the mothers had their first sexual intercourse was 17 years old. The association of postpartum age and her first sexual intercourse age $(p<0.001)$ identified earlier onset of sexual activity among adolescents. Conclusion: in general, they have inadequate knowledge of the fertile period, in addition to limited knowledge about contraceptive methods. There is a need for greater attention to family planning and care.
\end{abstract}

Descriptors: Pregnancy in adolescence; Sexual behavior; Reproductive history; Family planning; Contraception.

\section{RESUMEN}

Objetivo: describir y comparar los aspectos socio-demográficos, la historia sexual y reproductiva y el conocimiento anticonceptivo de las madres adolescentes y adultos que tenían sus entregas en una maternidad de referencia en la macro-región de salud. Métodos: fueron realizadas entrevistas individuales con instrumentos semi-estructurados con las cuestiones relativas a los aspectos estudiados. Resultados: La edad media de las madres fue de 26,74 años. Entre los métodos anticonceptivos, la píldora ha sido nombrado el más conocido. Se observó una asociación estadísticamente significativa entre el período fértil y la edad puerperal y los ingresos. La edad media a la que las madres tuvieron su primera relación sexual fue a los 17 años. La asociación edad puerperal y la edad de la primera relación sexual $(p<0,001)$ identificó inicio más temprano de la actividad sexual entre los adolescentes. Conclusión: las madres, generalmente, tienen un conocimiento inadecuado del período fértil, además de un conocimiento limitado acerca de los métodos anticonceptivos. Existe la necesidad de una mayor atención a la ayuda y a la planificación familiar.

Descriptores: Embarazo en adolescencia; Conducta sexual; Historia reproductiva; Planificación familiar; Anticoncepción.

${ }^{1}$ Graduada em Enfermagem. Doutora em Saúde Pública pela Universidade Federal de Minas Gerais. Docente na Universidade Federal dos Vales do Jequitinhonha e Mucuri. ${ }^{2}$ Graduada em Enfermagem pela Universidade Federal dos Vales do Jequitinhonha e Mucuri. ${ }^{3}$ Graduando em Enfermagem pela Universidade Federal dos Vales do Jequitinhonha e Mucuri. ${ }^{4} \mathrm{Graduada}$ em Enfermagem. Mestrado Profissional em Saúde, Sociedade e Ambiente pela Universidade Federal dos Vales do Jequitinhonha e Mucuri. ${ }^{5}$ Graduada em Enfermagem. Doutora em Enfermagem pela Universidade Federal de Minas Gerais. Docente na Universidade Federal dos Vales do Jequitinhonha e Mucuri. 


\section{INTRODUÇÃO}

As transformações culturais ocorridas na sociedade ao longo dos tempos contribuem cada vez mais para a assimilação de novas atitudes e valores frente às questões de sexualidade, influenciando, assim, o comportamento das mulheres adolescentes e adultas ${ }^{(1)}$. Os adolescentes têm iniciado precocemente a vida sexual e reprodutiva e, considerando as repercussões de uma gravidez não planejada para o futuro do jovem, essa questão merece destaque e planejamento por parte dos serviços de saúde ${ }^{(1-2)}$.

Direitos sexuais e reprodutivos, bem como a promoção da saúde de mulheres (adolescentes e adultas) são pautas de discussão da Organização das Nações Unidas (ONU) desde a década de 40. Inicialmente, como política de controle e, posteriormente, como forma de acessar tais direitos ${ }^{(3)}$. Esse tema também tem sido objeto de estudo na área da saúde coletiva, abrangendo questões como: características sociodemográficas, práticas sexuais, gravidez, doenças sexualmente transmissíveis, contracepção, aborto e violência ${ }^{(4)}$.

Mesmo com apoio de órgãos internacionais e acadêmicos, o Brasil ainda apresenta um cenário de fragilidade na implementação da legislação, déficit no conhecimento dos aspectos éticos, e legais por parte dos profissionais de saúde e baixo nível de informação dos adolescentes sobre a saúde sexual e reprodutiva ${ }^{(3)}$.

Nesse sentido, a gravidez precoce e não planejada é considerada um problema de saúde pública no Brasil( ${ }^{(1)}$. Independente da faixa etária, é importante ressaltar que o planejamento familiar precisa realizar ações direcionadas à saúde integral da mulher, condizendo com as ações realizadas pela Estratégia de Saúde da Família.

Os serviços de saúde devem proporcionar atendimento a adolescentes e jovens, antes mesmo do início de sua atividade sexual reprodutiva, instigando práticas de prevenção e autocuidado $^{(1)}$. Entretanto, sabe-se que o planejamento familiar preconizado pelo Ministério da Saúde oferece maior ênfase ao ciclo gravídico-puerperal ${ }^{(2)}$.

Em 2009, estimou-se que, aproximadamente, $20 \%$ de gestantes no Brasil eram adolescentes, ou seja, há, em média, uma adolescente entre cada cinco mulheres grávidas ${ }^{(2)}$. Em 2014, a incidência de gravidez entre adolescentes no país foi de $18,9 \%$ dos nascidos vivos. O estado de Minas Gerais apresentou $16,0 \%$ e a macrorregião de saúde do Jequitinhonha seguiu a tendência nacional com, aproximadamente, $18,6 \%$ de partos em adolescentes $^{(5)}$.

Diante do exposto, a realização dessa pesquisa justifica-se pelo fato de a ocorrência da gravidez precoce acarretar várias consequências para a futura mãe, familiares e o recém-nascido.

Nesse sentido, o estudo comparativo do perfil epidemiológico entre as puérperas adolescentes e adultas, considerando o perfil socioeconômico, a história reprodutiva, comportamento sexual e contraceptivo, pode contribuir diretamente para o planejamento das ações, principalmente de enfermagem, voltadas para a mulher, criança e adolescente. A partir da identificação das fragilidades existentes em cada grupo etário, é possível fortalecer as políticas públicas de saúde da família.

Assim, esse trabalho tem por objetivo descrever e comparar os aspectos sociodemográficos, a história sexual e reprodutiva e o conhecimento contraceptivo de puérperas adolescentes e adultas que tiveram seus partos numa maternidade de referência para a macrorregião de saúde de Diamantina-MG.

\section{MÉTODOS}

Trata-se de um estudo do tipo transversal, descritivo e como público-alvo, puérperas adolescentes (sendo consideradas, segundo a OMS, mulheres com idade entre 10 e 19 anos) ${ }^{(6)} \mathrm{e}$ adultas que tiveram seus partos numa maternidade de referência para a macrorregião de saúde de Diamantina - MG e com maior número de nascimentos/ano. Este estudo foi apreciado e aprovado pelo Comitê de Ética e Pesquisa da Universidade Federal dos Vales do Jequitinhonha e do Mucuri - CEP/UFVJM sob o parecer número 105/12.

A seleção da amostra, inicialmente, realizou-se pelo levantamento no banco de dados oficial, DATASUS, sobre o número de partos ocorridos no município de Diamantina. O último período informado foi o ano de 2010, contabilizando 1295 partos $^{(5)}$. Foi realizado, em um segundo momento, uma amostragem aleatória simples considerando o intervalo de confiança de $95 \%$ e ponderando as possíveis perdas, chegando ao total de 327 puérperas que, posteriormente, foram entrevistadas.

Foram critérios de inclusão dos sujeitos, independente da faixa etária, aceitar participar da pesquisa, ter o parto realizado na maternidade do município de Diamantina e estar em período de internação na referida maternidade. Foram 
excluídas as mães que estavam em condição de intercorrência ou complicação pós-parto que impossibilitavam a participação e as portadoras de surdez ou mudez.

Para a coleta de dados, foi utilizado um instrumento semiestruturado contendo questões relativas aos aspectos socioeconômicos, demográficos, história reprodutiva (idade da menarca, idade da primeira relação sexual, idade da primeira gestação, aborto), comportamento sexual e contraceptivo (conhecimento sobre o período fértil, conhecimento e uso de método contraceptivo). Todas as variáveis foram mensuradas por respostas espontâneas, ou seja, não houve leitura de alternativas de respostas.

As entrevistas ocorreram no período de maio de 2013 a março de 2014, no local de internação da entrevistada, ou seja, enfermaria ou apartamento. Foi realizado, anteriormente, o teste-piloto para a adequação do instrumento de coleta de dados. Entrevistaram-se duas puérperas por dia, considerando o período de segunda a sexta-feira, sendo selecionadas as mulheres que tiverem os dois primeiros partos do dia. Cada uma delas foi convidada a assinar um Termo de Consentimento Livre e Esclarecido (TCLE). As puérperas adolescentes explicitaram sua anuência em participar da pesquisa através de um Termo de Assentimento (TA), além do TCLE assinado pelo seu responsável.
Foi elaborado um banco de dados no programa estatístico Statistical Package for Social Science (SPSS), versão 20.0, para armazenar as informações obtidas por meio das entrevistas. Posteriormente, após verificação de possíveis inconsistências, os dados foram analisados utilizando-se os testes estatísticos de Teste de Mann-Whitney, Teste de Kruskal-Wallis e Teste Qui-Quadrado. Posteriormente, os dados foram discutidos com base na literatura nacional e internacional.

\section{RESULTADOS E DISCUSSÃO}

Foram entrevistadas, ao longo da pesquisa, 327 puérperas. Foi predominante o número de puérperas adultas, com uma amostra de 255 (78\%), e 72 (22\%) adolescentes (entre 10 e 19 anos de idade). A média de suas idades foi de 26,7 anos, com um desvio padrão de 6,82, observando extremos de 15 e 45 anos.

A Tabela 1 descreve as características demográficas e socioeconômicas das puérperas entrevistadas.

Os resultados mostram que as mães adultas tendem a ser casadas ou morar junto com o marido ou companheiro há mais de um ano em relação às mães adolescentes. Com relação à escolaridade, aproximadamente dois terços das puérperas possuem ensino médio ou superior, não tendo, esse dado, relação com a idade materna nessa amostra.

Tabela 1 - Características demográficas e socioeconômicas das puérperas que tiveram seus partos em uma maternidade de referência para a macrorregião de saúde de Diamantina-MG, maio de 2013 a março de 2014.

\begin{tabular}{|c|c|c|c|}
\hline Variável & $\begin{array}{c}\text { Mães adolescentes } \\
\mathrm{n}(\%)\end{array}$ & $\begin{array}{c}\text { Mães adultas } \\
\text { n (\%) }\end{array}$ & $\begin{array}{l}\text { Valor } \\
\text { de } p\end{array}$ \\
\hline \multicolumn{4}{|l|}{ Estado civil } \\
\hline Casado, mora junto & $40(55,6)$ & $210(82,4)$ & \\
\hline Solteiro, separado, divorciado & $32(44,40)$ & $45(17,6)$ & $<0,001$ \\
\hline \multicolumn{4}{|l|}{ Tempo de casada } \\
\hline Até um ano & $18(45,0)$ & $37(17,6)$ & \\
\hline Mais que 1 ano & $22(55,0)$ & $173(82,4)$ & $<0,001$ \\
\hline \multicolumn{4}{|l|}{ Escolaridade } \\
\hline Até ensino fundamental & $24(33,3)$ & $87(34,1)$ & \\
\hline Ensino médio ou superior & $48(66,7)$ & $168(65,9)$ & 0,901 \\
\hline \multicolumn{4}{|c|}{ Com quem morava quando engravidou } \\
\hline Marido, companheiro & $19(26,4)$ & $172(67,5)$ & \\
\hline Familiares & $42(58,3)$ & $54(21,2)$ & \\
\hline Amigo, sozinha & $1(1,4)$ & $8(3,1)$ & \\
\hline Marido, familiares & $10(13,9)$ & $20(7,8)$ & \\
\hline Outros & $0(0,0)$ & $1(0,4)$ & $<0,001$ \\
\hline
\end{tabular}

Fonte: Elaborada pelos autores.

Estudos realizados no Brasil e na Inglaterra revelam que, nos últimos anos, vem sendo observada uma redução nas taxas de gestação na adolescência. Contudo, essa redução não ocorre 
de maneira uniforme, mas apresenta desigualdades, de acordo com o desenvolvimento social do território, sendo menor nas classes sociais mais excluídas ${ }^{(7)}$.

Pesquisa realizada em uma grande cidade do estado de São Paulo apontou que a maioria das adolescentes grávidas coabitavam com o companheiro ou eram solteiras e somente $10 \%$ casadas $^{(8)}$. Já uma pesquisa feita na Escócia revelou que o status socioeconômico também foi altamente associado com a gravidez na adolescência, uma vez que as adolescentes possuíam pior nível de escolaridade e renda familiar, porém viviam mais comumente sem companheiro $^{(9)}$.

Observou-se que a maioria das puérperas $(82,6 \%)$ teve sua primeira menstruação com idade entre 11 e 15 anos, tendo como média a idade de 12 anos. No que diz respeito à gestação, $60,6 \%$ das entrevistadas afirmaram que essa não foi a sua primeira gestação. Dentre elas, a maioria teve apenas uma gestação antes da atual, tendo, como média 1,23 filhos e mediana 1.

A idade da menarca é um sinal do começo da fase reprodutiva da mulher, sendo considerado um importante preditor da saúde na adolescência, ocorrendo, em média, por volta dos 12 anos de idade. Ocorre, após a menarca, uma grande maturação uterina que permite o acontecimento da gravidez ${ }^{(10)}$. Os profissionais de saúde devem se preocupar com o início da vida sexual na adolescência porque esse evento pode colocar o adolescente em situação de vulnerabilidade às doenças sexualmente transmissíveis, gestação não planejada e aborto ${ }^{(11)}$.

A mediana das idades em que as puérperas ficaram grávidas pela primeira vez foi de 20 anos, tendo como extremos, idades de 13 e 43 anos. A média de número de filhos tidos vivos pelas puérperas foi de 2 .

A tabela 2 descreve algumas variáveis categóricas relacionadas à história reprodutiva das puérperas de acordo com a idade. As respostas revelaram, com significância estatística, que a maior parte das adolescentes estavam em sua primeira gestação $(76,4 \%)$ e que quase a totalidade das adolescentes $(97,2 \%)$ não havia passado pela experiência de aborto prévio. Entre as adultas, $21,2 \%$ já tiveram histórico de aborto natural ou provocado.

Tabela 2. História reprodutiva de acordo com a idade das puérperas que tiveram seus partos em uma maternidade de referência para a macrorregião de saúde de Diamantina-MG, maio de 2013 a março de 2014.

\begin{tabular}{cccc}
\hline $\begin{array}{l}\text { Variável } \\
\text { Menarca }\end{array}$ & Mães adolescentes $\mathbf{n}(\%)$ & Mães adultas $\mathbf{n}(\%)$ & Valor de $\mathbf{p}$ \\
Menor que 11 anos & $7(9,7 \%)$ & $13(5,1 \%)$ & \\
11 anos ou mais & $64(88,9 \%)$ & $239(93,7 \%)$ & $0,16^{*}$ \\
Não sabe & $1(1,4 \%)$ & $3(1,2 \%)$ & \\
Primeira gestação & & & \\
Sim & $55(76,4 \%)$ & $74(29 \%)$ & $<0,001^{* *}$ \\
Não & $17(23,6 \%)$ & $181(71 \%)$ & \\
Aborto prévio & & & $<0,001^{* *}$ \\
$\quad$ Sim & $2(2,8 \%)$ & $54(21,2 \%)$ & \\
Não & $70(97,2 \%)$ & $201(78,8 \%)$ & $0,86^{*}$ \\
Aborto espontâneo*** & $2(100,0 \%)$ & $50(92,6 \%)$ & \\
Sim & $0(0 \%)$ & $4(7,4 \%)$ & \\
Não & de 20 anos & $152(46,5 \%)$ & $0,113^{* *}$ \\
Sua mãe teve o primeiro filho com menos & $41(56,9 \%)$ & $118(36,1 \%)$ & \\
Sim & $22(30,6 \%)$ & $57(17,4 \%)$ & \\
Não & $9(12,5 \%)$ &
\end{tabular}

Fonte: Elaborada pelos autores.

*Teste Exato de Fisher. ${ }^{* *}$ Teste Qui-Quadrado.

***Análise realizada apenas com aquelas puérperas que relataram aborto, total 56 .

As adolescentes que vivem em condições socioeconômicas mais vulneráveis apresentam maior número de gestações, parceiros sexuais, casos de abortamento e histórico de violência sexual ${ }^{(10)}$. A reincidência da gravidez até dois anos após o término de uma gestação ocorrida 
durante a adolescência está associada com morar no interior, abandono dos estudos, baixa escolaridade, renda familiar de até um salário mínimo e dependência financeira ${ }^{(12)}$. Mais de $50 \%$ das adolescentes no presente estudo provêm de famílias nas quais a gestação na adolescência é uma experiência comum, ocorrência menor que a descrita em outro estudo ${ }^{(8)}$. Estudo realizado no Canadá apontou como as relações familiares podem influenciar na gestação na adolescência. 0 fato de ter uma irmã que engravidou na adolescência ou uma mãe que teve filho antes dos 20 anos são preditores de gravidez nessa faixa etária(13).

No Brasil, o aborto destaca-se como um grave problema de saúde pública à medida que é amplamente praticado, muitas vezes de forma insegura, dentro de um cenário de clandestinidade. O abortamento espontâneo também é frequente e mostrado pelas mulheres como um momento difícil, triste e doloroso, em que ela exprime ansiedade, medo e insegurança ${ }^{(14)}$. Além disso, o processo de aborto coloca em risco a vida das mulheres, expondo-as a complicações que podem ter um impacto sobre sua saúde no contexto biopsicossocial. Um estudo realizado numa Universidade de Washington revelou uma redução clínica e estatisticamente significativa nas taxas de aborto $^{(15)}$.

O Ministério da Saúde criou, no início dos anos 80, o Programa de Atenção Integral à Saúde da Mulher (PAISM), que contribuiu para a inserção de uma nova abordagem à saúde da mulher, incluindo, dentre suas ações, questões relacionadas ao planejamento familiar, adotando medidas e políticas para permitir o acesso da população aos meios de contracepção ${ }^{(2)}$.

A Tabela 3 mostra a descrição do conhecimento de métodos contraceptivos pelas puérperas. A pílula foi o método mais referido $(96,3 \%)$, seguido do preservativo $(87,8 \%)$ e do DIU (53,2\%). O espermaticida foi o método menos citado, uma vez que apenas duas puérperas afirmaram ter conhecimento $(0,2 \%)$. Vale ressaltar que, dentre as 327 entrevistadas, 8 $(2,4 \%)$ referiram não conhecer nenhum método contraceptivo.

Tabela 3 - Conhecimento de métodos contraceptivos pelas puérperas que tiveram seus partos em uma maternidade de referência para a macrorregião de saúde de Diamantina-MG, maio de 2013 a março de 2014.

\begin{tabular}{|c|c|c|c|}
\hline Método contraceptivo & Adolescente N (\%) & Adulta N (\%) & Valor de $P$ \\
\hline Pílula & $66(91,7)$ & $249(97,6)$ & $0,028^{*}$ \\
\hline Preservativo & $58(80,6)$ & $229(89,8)$ & $0,034^{* *}$ \\
\hline DIU & $19(26,4)$ & $155(60,8)$ & $<0,001^{* *}$ \\
\hline Anticoncepcional injetável & $26(36,1)$ & $67(26,3)$ & $0,102 * *$ \\
\hline Ligadura & $5(6,9)$ & $30(11,8)$ & $0,243^{* *}$ \\
\hline Tabelinha & $0(0,0)$ & $32(12,5)$ & $0,002 * *$ \\
\hline Diafragma & $4(5,6)$ & $28(11,0)$ & $0,171^{* *}$ \\
\hline Vasectomia & $1(1,4)$ & $10(3,9)$ & $0,467^{*}$ \\
\hline Coito interrompido & $1(1,4)$ & $9(3,5)$ & $0,697^{*}$ \\
\hline Espermaticida & $0(0,0)$ & $2(0,8)$ & $1,000 *$ \\
\hline
\end{tabular}

Fonte: Elaborada pelos autores.

*Teste Exato de Fisher. **Teste Qui-Quadrado.

Ressalta-se que houve associação estatisticamente significante entre conhecimento e uso de método contraceptivo e série escolar ( $p=0,026$ e $p=0,003$, respectivamente), ou seja, as puérperas que tem maior conhecimento acerca de métodos contraceptivos são aquelas que mais fazem uso dos mesmos e que têm maior escolaridade (ensino médio ou superior).

Com relação ao uso de algum método contraceptivo na época em que engravidou, a maioria $(62,4 \%)$ afirmou não ter feito uso de nenhum método. Das que utilizaram $(37,6 \%)$, a sequência dos métodos mais usados foi: pílula $(22,3 \%)$, seguido do preservativo $(10,1 \%)$, anticoncepcional injetável (1,5\%), DIU $(1,2 \%)$, tabelinha $(0,9 \%)$, pílula e preservativo $(0,9 \%)$ e pílula e anticoncepcional injetável $(0,3 \%)$, sendo que, dentre elas, uma não quis informar qual método estava utilizando. Os métodos relatados como de conhecimento das puérperas desse estudo coincidem com um estudo realizado em Teresina, Piauí ${ }^{(16)}$.

A anticoncepção tem adquirido papel importante na saúde reprodutiva e seu uso, de 
forma inadequada, implica agravos à saúde da mulher, como gravidez indesejada, gravidez na adolescência, abortamentos ilegais, adoecimento por infecções sexualmente transmissíveis e até mesmo aumento na mortalidade materna. Da mesma forma, apontados nesse estudo, outros trabalhos mostram que os principais métodos contraceptivos conhecidos e utilizados pelas mulheres, inclusive as adolescentes, são a pílula e o preservativo masculino e feminino ${ }^{(8,16-18)}$. Outro ponto importante relacionado a esses métodos diz respeito ao uso duplo dos mesmos, uma vez que muitas mulheres preocupam-se apenas em evitar a gravidez, esquecendo-se dos métodos que evitam doenças. Assim, é de suma importância a dupla proteção (uso do preservativo e de outro método contraceptivo, como o contraceptivo oral, por exemplo) ${ }^{(10,19)}$.

Um aspecto importante observado nessa pesquisa, que corrobora outras pesquisas sobre $o$ tema, é que as mulheres, em especial as adolescentes, possuem conhecimento limitado e/ou inadequado sobre os métodos contraceptivos, bem como sobre seu período fértil ${ }^{(1,16,20-21)}$. Além disso, informações obtidas de forma inadequada e uso de contraceptivos sem a correta orientação técnica podem comprometer a eficácia do método ${ }^{(10)}$.

Porém, o não uso de pelo menos um método contraceptivo não pode ser atribuído somente ao desconhecimento da importância e da forma de utilização, mas também ao não planejamento das relações, ao desejo de engravidar e medo de magoar o parceiro ${ }^{(20,22)}$.

Dentre as puérperas que não utilizavam nenhum método na época em que engravidaram $(n=204)$, a maioria (54\%) referiu desejo de engravidar, justificativa que também foi a mais prevalente em outro estudo, mostrando que as motivações pessoais possuem fortes contribuições para a maior ou menor utilização dos métodos contraceptivos ${ }^{(16)}$. Ademais, participantes dessa pesquisa revelaram outros motivos para a não utilização de algum método contraceptivo, tais como esquecimento /desinteresse (22\%), intolerância aos anticoncepcionais $(10 \%)$, o parceiro não quis $(5 \%)$, falta de informação, impossibilidade financeira e outro (5\%).

Um estudo com amostra nacional de adolescentes estudantes revelou que o uso de contraceptivo é influenciado pelas características sociodemográficas, sendo maior entre mulheres, na fase mais avançada da adolescência, de escola privada e residentes na região sul do país ${ }^{(11)}$.

Observou-se, na última década, um aumento do conhecimento e uso de métodos contraceptivos entre mulheres de 15 a 24 anos, que pode estar relacionado aos aspectos como escolaridade e nível socioeconômico ${ }^{(20)}$. No presente trabalho, as fontes de informação, a respeito do uso de métodos contraceptivos, citadas pelas mulheres entrevistadas foram a escola $(42,2 \%)$ e os profissionais de saúde $(22,3 \%)$. Fontes como o companheiro/parceiro sexual e a mídia não apresentaram relevância. Ao contrário de um estudo feito na Etiópia, em que as principais fontes de informação sobre tais métodos foram a televisão e o rádio ${ }^{(23)}$, sugerindo uma forma de abordagem do tema a ser considerada no cenário estudado.

Com relação ao período fértil, $75,2 \%$ das puérperas afirmaram não ter conhecimento sobre o seu período, sendo que a maioria das que diziam conhecer não tinham conhecimento devidamente correto. Houve associação estatisticamente significante entre o conhecimento do período fértil e a idade da puérpera $(p=0,006)$ e a renda $(p=0,02)$, comprovando que as adultas e as puérperas com maior renda, são aquelas com maior conhecimento acerca do seu período fértil. Um estudo de inquérito domiciliar com adolescentes e adultas revelou conhecimento do período fértil por apenas $20 \%$ de uma amostra com mais de 600 jovens $^{(20)}$.

A mediana de idade em que as puérperas tiveram sua primeira relação sexual foi de 17 anos, tendo como extremos as idades de 12 e 30 anos. O uso de algum método contraceptivo na primeira relação foi referido por 192 puérperas (58,7\%), enquanto 106 (32,4\%) afirmaram não ter usado nenhum método, 14 (4,3\%) não sabiam afirmar e 15 (4,6\%) não quiseram falar a respeito. Houve associação estatisticamente significativa entre idade da puérpera e idade da primeira relação sexual $(p<0,001)$, comprovando que as puérperas adolescentes iniciaram a vida sexual mais cedo que as adultas. A idade da primeira relação corroborou com um estudo realizado com adolescentes em uma capital do nordeste que associaram, significativamente, ao início da vida sexual, a escolaridade, a idade da menarca, o tempo de namoro com o primeiro parceiro sexual e a orientação sexual da escola ${ }^{(24)}$.

$\mathrm{Na}$ Tabela 4, tem-se a associação entre idade da primeira relação sexual e idade da 
menarca estratificada pela idade da puérpera. Estes resultados mostram que, dentre as adolescentes, houve associação entre idade da primeira relação e idade da menarca $(p=0,040)$; pelos dados, pode-se afirmar que as adolescentes que tiveram menarca mais "cedo" (menos de 11 anos) iniciaram a vida sexual precocemente; entretanto, não houve correlação estatisticamente significante entre idade da primeira relação e idade da menarca para as adultas.

Tabela 4 - Associação entre idade da menarca e idade da primeira relação sexual de acordo com a idade das puérperas que tiveram seus partos em uma maternidade de referência para a macrorregião de saúde de Diamantina-MG, maio de 2013 a março de 2014.

Idade primeira relação sexual Média (DP)

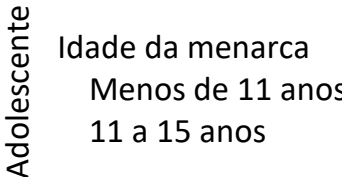

Idade da menarca

$\begin{array}{ll}\frac{\pi}{5} & \text { Menos de } 11 \text { anos } \\ \frac{ \pm}{5} & 11 \text { a } 15 \text { anos }\end{array}$

Mais de 15 anos
$13,75(1,7)$

$15,56(1,7)$

$0,040^{*}$

$17,30(3,36)$

$17,74(2,86)$

$18,64(3,24)$

P

\section{.}

Fonte: Elaborada pelos autores.

*Teste de Mann-Whitney.

**Teste de Kruskal-Wallis.

As transformações culturais ocorridas em nossa sociedade têm contribuído cada vez mais para a assimilação de novas atitudes e valores frente às questões de sexualidade, interferindo no comportamento dos adolescentes, fazendo com que a primeira relação sexual ocorra cada vez mais cedo ${ }^{(1-2)}$.

Pesquisas revelam uma média de idade de início de atividade sexual de 15 anos, variando entre estudos(11,20,25). Em relação ao uso de métodos contraceptivos na primeira relação, um estudo realizado no Distrito Federal apontou que $54 \%$ das puérperas não utilizaram nenhum método na primeira relação sexual e $44 \%$ alegaram ter usado algum método(1). Quanto mais cedo ocorre a primeira relação sexual, menor a chance de utilizar um método contraceptivo(25). A iniciação sexual, por si só, não significa comportamento de risco, mas introduz o jovem em uma parcela da população considerada susceptível para as doenças sexualmente transmissíveis. O uso inconstante do preservativo ainda é muito prevalente entre adolescentes ${ }^{(22)}$.

Quanto aos temas relacionados à idade da mãe da puérpera quando teve seu primeiro filho e o número de filhos tidos vivos e mortos pela puérpera ao longo de sua vida, pondera-se o indício de que a história obstétrica se repete por gerações, uma vez que a família exerce forte influência na saúde da mulher ${ }^{(12)}$.

\section{CONCLUSÃO}

Ao final da pesquisa, foi possível concluir que as puérperas, tanto as adolescentes quanto as adultas, em sua maioria, não possuem conhecimento do período fértil, além do conhecimento limitado acerca dos métodos contraceptivos. A escola e os profissionais de saúde foram as principais fontes de tal informação, porém a mídia não mostrou relevância.

O caráter regional desse estudo pode ser considerado como uma limitação, uma vez que se trata de uma maternidade de menor porte, o que resultou em um menor número amostral. Entretanto, evidencia-se que, embora as necessidades em saúde reprodutiva das mulheres adultas possam se divergir das adolescentes, os resultados confirmam a necessidade de maior atenção à assistência e ao planejamento familiar. Esse achado mostra a importância de melhorias no desempenho do nosso sistema de saúde no que se refere à informação das mulheres (adolescentes e adultas) sobre o uso correto de contraceptivos e à ênfase na continuidade da aplicação do método de escolha. Fazendo-se, também necessária, a promoção de programas que, efetivamente respeitem os direitos sexuais e reprodutivos dos adolescentes. 
É possível, a partir desse estudo, propor nova pesquisa de cunho qualitativo para ampliar a compreensão de questões peculiares da vida sexual e reprodutiva e subsidiar a promoção à saúde na Atenção Básica da macrorregião, uma vez que os estudos quantitativos não possibilitam o conhecimento profundo de todas as dimensões que envolvem comportamentos e fenômenos da vida humana. É importante abordar aspectos como: planejamento familiar, fisiologia do aparelho ginecológico feminino, o conhecimento sobre contraceptivos e outras questões pessoais e familiares que envolvem maternidade em várias fases da vida da mulher.

\section{REFERÊNCIAS BIBLIOGRÁFICAS}

1 - Duarte CF, Holanda LB, Medeiros ML. Avaliação de conhecimento contraceptivo entre adolescentes grávidas em uma unidade básica de saúde do Distrito Federal. J Health Sci Inst. 2012 [citado em 8 jun 2015]; 20(2):140-43. Disponível em:

https://www.unip.br/presencial/comunicacao/pu blicacoes/ics/edicoes/2012/02 abr-jun/V30 n2 2012 p140-143.pdf

2 - Moura LNB, Gomes KRO. Planejamento familiar: uso dos serviços de saúde por jovens com experiência de gravidez. Ciênc Saúde Coletiva 2014;19(3):853-63. DOI: 10.1590/ 141381 232014193.10902013

3 - Moraes SP, Vitalle MSS. Direitos sexuais e reprodutivos na adolescência: interações ONUBrasil. Ciênc Saúde Coletiva 2015;20(8):2523-31. DOI: 10.1590/1413-81232015208.03112014

4 - Milne FH, Judge DS. A novel quantitative approach to women's reproductive strategies. PLoS One 2012;7(10):1-11. DOI: 10.1371/journal. pone. 0046760

5 - Ministério da Saúde. Sistema de nascidos vivos. Brasília: Ministério da Saúde; 2016 [citado em 8 jan 2016]. Available in: http://www2.datasus.gov.br/DATASUS/index.php ?area $=0205$

6 - World Health Organization (WHO). Adolescent pregnancy: fact sheet. Washington: WHO; 2016 [cited in 2017 Feb 04]. Available in: http://www.who.int/mediacentre/factsheets/fs3 64/en/index.html

7 - Girma S, Paton D. Is education the best contraception: The case of teenage pregnancy in England? Soc Sci Med. 2015;131:1-9. DOI: 10.1016/i.socscimed.2015.02.040

8 - Vieira EM, Bousquat A, Barros CRDS, Alves MCGP. Adolescent pregnancy and transition to adulthood in young users of the SUS. Rev Saúde Pública 2017;51:1-11. DOI: 10.1590/S1518-8787. 2017051006528

9 - Mccall SJ, Bhattacharya S, Okpo E, Macfarlane GJ. Evaluating the social determinants of teenage pregnancy: A temporal analysis using a UK obstetric database from 1950 to 2010. J Epidemiol Community Health 2015;69(1):49-54. DOI: 10.1136/iech-2014-204214

10 - Pereira SM, Taquette SR, Pérez MA. Consulta ginecológica sob a ótica de estudantes do ensino médio do Rio de Janeiro, RJ. Rev Saúde Pública 2013;47(1):2-10. DOI: 10.1590/S0034-89102013 000100002

11 - Borges ALV, Fujimori E, Kuschnir MCC, Chofakian CBN, Moraes AJP, Azevedo GD, et al. ERICA: sexual initiation and contraception in Brazilian adolescents. Rev Saúde Pública 2016;50(supl 1):1-11. DOI: 10.1590/S01518$\underline{8787.2016050006686}$

12 - Nery IS, Gomes KRO, Barros IC, Gomes IS, Fernandes ACN, Viana LMM. Fatores associados à reincidência de gravidez após gestação na adolescência no Piauí, Brasil. Epidemiol Serv Saúde 2015;24(4):671-80. DOI: 10.5123/S167949742015000400009

13 - Wall-Wieler E, Roos LL, Nickel NC. Teenage pregnancy: The impact of maternal adolescent childbearing and older sister's teenage pregnancy on a younger sister. BMC Pregnancy Childbirth 2016;16:120. DOI: 10.1186/s12884-016-0911-2

14 - Domingos SRF, Merighi MAB, Jesus MCP. Experience and care in miscarriage: $A$ qualitative study. Online Braz J Nurs. 2011;10(2):1-9. DOI: 10.5935/1676-4285.20113236

15 - Peipert JF, Madden T, Allsworth JE, Secura GM. Preventing unintended pregnancies by providing no-cost contraception. Obstet Gynecol. 2012;120(6):1291-7. DOI: 10.1097/AOG.0b013e 318273eb56

16 - Araújo AKL, Nery IS. Knowledge about contraception and factors associated with pregnancy planning in adolescence. Cogitare Enferm. 2018;23(2):1-10. DOI: 10.5380/ce v23i2. $\underline{55841}$

17 - Tran N, Stapleton J, Zhang Y, Harlow S, Yang $\mathrm{H}$, Moyer CA. Contraceptive practices of women visiting a gynecology clinic in Beijing, China. Int J Gynaecol Obstet. 2011;112(1):64-5. DOI: 10.1016/j.ijgo.2010.08.003

18 - Ritter T, Dore A, Mcgeechan K. Contraceptive knowledge and attitudes among 14-24-year-olds in New South Wales, Australia. Aust N Z J Public 
Health 2015;39(3):267-9. DOI: 10.1111/1753$\underline{6405.12367}$

19 - Lopez LM, Grey TW, Chen M, Hiller JE. Behavioral interventions for improving dualmethod contraceptive use. Cochrane Database Syst Rev. 2014;30(3):1-38. DOI: 10.1002/14651858.CD010915

20 - Olsen JM, Lago TDG, Kalckmann S, Alves MCGP, Escuder MML. Young women's contraceptive practices: A household survey in the city of São Paulo, Brazil. Cad Saúde Pública 2018;34(2):e00019617. DOI: 10.1590/0102311x00019617

21 - Bunting L, Tsibulsky I, Boivin J. Fertility knowledge and beliefs about fertility treatment: Findings from the International Fertility Decisionmaking Study. Hum Reprod. 2013;28(2):385-97. DOI: $10.1093 /$ humrep/des402

22 - Soares LR, Cabero FV, Souto TG, Coelho RFS, Lacerda LCM, Matão MEL. Assessment of sexual behavior among young people and adolescents at government schools. Adolesc Saúde 2015 [citado em 8 jun 2015]; 12(2):76-84. Disponível em: http://www.adolescenciaesaude.com/detalhe ar tigo.asp?id=504\&idioma $=$ English

23 - Melaku YA, Berhane Y, Kinsman J, Reda HL. Sexual and reproductive health communication and awareness of contraceptive methods among secondary school female students, northern Ethiopia: a cross-sectional study. BMC Public Health 2014;14(252):1-11. DOI: 10.1186/14712458-14-252

24 - Maranhão TA, Gomes KRO, Oliveira DC, Moita Neto JM. Repercussão da iniciação sexual na vida sexual e reprodutiva de jovens de capital do Nordeste brasileiro. Ciênc Saúde Coletiva 2017;22(12):4083-94. DOI: 10.1590/1413812320172212.16232015

25 - Ramos ACP, Amaral JG, Rodarte AC, Costa MA, Oliveira PP. Consulta ginecológica sob a ótica de adolescentes. $R$ Enferm Cent $O$ Min. 2014;3(4):1248-61. DOI: $10.19175 /$ recom.v $\underline{0 \mathrm{i} 0.755}$

Nota: Este trabalho não foi financiado por órgãos de fomento e faz parte de Monografia final de Curso de Graduação em Enfermagem.

Recebido em: 21/03/2018

Aprovado em: 22/08/2018

Endereço de correspondência:

Maristela Oliveira Lara

Campus JK - Diamantina/MG
Rodovia MGT 367- Km 583, no 5000

Alto da Jacuba

CEP: 39.100-000 Brasil

E-mail: maryslara@hotmail.com 